\title{
La empatía y el apoyo de la pareja como factores protectores del estrés en pacientes con cáncer
}

\section{Empathy and partner support as a protective factors of stress in cancer patients}

\author{
Donaji Zedith Santiago-Silva y Rozzana Sánchez-Aragón
}

\author{
Universidad Nacional Autónoma de México ${ }^{1}$
}

Autor para correspondencia: Rozzana Sánchez Aragón, rozzara@unam.mx.

\begin{abstract}
RESUMEN
En México, el cáncer representa un grave problema de salud pública. Debido a esta enfermedad, el paciente enfrenta grandes cambios emocionales, conductuales y sociales que generan un considerable estrés emocional; por ello, su pareja asume un papel vital para aminorarlos y facilitar así el apoyo social y psicológico requerido por aquel. En consecuencia, el presente estudio tuvo como objetivos examinar los efectos que tiene la empatía de la pareja en el paciente oncológico, así como la satisfacción del paciente con dicho apoyo y con su capacidad para recibirlo. Participaron 194 parejas heterosexuales en las que uno de sus miembros tenía un diagnóstico de cáncer, quienes respondieron de manera voluntaria diversas escalas: Empatía en el Contexto Romántico, Satisfacción con el Apoyo recibido de la Pareja, Disposición a recibir Apoyo Social y Estrés Percibido. Los hallazgos mostraron relaciones significativas positivas entre la empatía del hombre sano, la satisfacción con el apoyo y la capacidad de recibirlo en la mujer con cáncer y el control del estrés de esta última, así como efectos positivos y significativos de la satisfacción con el apoyo en momentos de necesidad y con la capacidad para recibirlo del hombre con cáncer en el control del estrés.
\end{abstract}

Palabras clave: Cáncer; Cuidados paliativos; Estrés; Apoyo social; Empatía; Sexo.

\begin{abstract}
In Mexico, cancer represents a public health problem, where the patient faces great emotional, behavioral and social changes, inciting emotional stress, therefore the couple takes a vital role when reduce it, since it facilitates access to social and psychological support. This research aimed to examine the effects of the empathy of the cancer patient's partner, as well as the satisfaction with the support and the ability to receive it from the cancer patient, on the stress of the latter, performing separate analyzes by gender. This work had the participation of 194 heterosexual couples, in which one member had a cancer diagnosis, who voluntarily responded to the Empathy in the Romantic Context, Satisfaction with the Support Received from the Partner, Willingness to Receive Social Support and Perceived Stress scales. The findings showed significant positive relationships between the empathy of the healthy man, satisfaction with support and the ability to receive it from the woman with cancer, with the control of stress in the latter. And on the other hand, positive and significant effects of satisfaction with support in times of need and the ability to receive it from the man with cancer on his own stress management.
\end{abstract}

Key words: Cancer; Palliative care; Stress; Social support; Empathy; Sex.

\footnotetext{
${ }^{1}$ Facultad de Psicología, Av. Universidad 3004, Col. Copilco Universidad, Coyoacán, 04510 Ciudad de México, México.
} 
$E^{1}$ 1 término cáncer engloba un grupo numeroso de enfermedades caracterizadas por el desarrollo de células anormales que se dividen, crecen y se diseminan sin control en cualquier parte del cuerpo (Puente y De Velasco, 2019), sobrepasando el número de las células normales, lo que dificulta que el cuerpo funcione de la manera apropiada (American Cancer Society, 2016).

Hay más de cien tipos de cáncer (INFO Cáncer, 2019), los que reciben por lo general el nombre de los órganos o tejidos en los que se forman (National Cancer Institute, 2015) y que muestran un distinto comportamiento, pronóstico y método de tratamiento (Puente y De Velasco, 2019). Esta enfermedad implica una carga enorme para los pacientes, las familias y la sociedad (Organización Mundial de la Salud [OMS], 2017) debido a que constituye un reto para el desarrollo socioeconómico, la sostenibilidad medioambiental, la reducción de la pobreza y el bienestar de los individuos (Organización Panamericana de la Salud [OPS] y OMS, 2013). El cáncer se ha posicionado como la segunda causa de muerte en el mundo en números tangibles (OMS, 2016, 2017, 2018). Específicamente en México, el cáncer es la tercera causa muerte y afecta a cualquier grupo de edad. Tan solo en el lapso de 2011 a 2016, tres hombres y dos mujeres de cada cien mil, de entre 18 y 29 años de edad, fallecieron por este padecimiento (Instituto Nacional de Estadística y Geografía [INEGI], 2018), cifras que aumentan según la edad de los enfermos.

Un aspecto muy importante a considerar en el tratamiento del cáncer son los denominados cuidados paliativos, los cuales tienen como objetivo aliviar - no curar- los síntomas y consisten en la atención que brinda el equipo de salud para ayudar a los enfermos a vivir más confortablemente, es decir, con una mejor calidad de vida (De la Garza y Juárez, 2014), favoreciendo así el bienestar en aspectos importantes para el individuo, ya sean físicos, psicológicos, sociales o espirituales. Para la OMS (2018), este tipo de cuidados son el segundo objetivo más importante en el tratamiento del cáncer, solo después de la cura o de la prolongación de la vida, porque bien empleados pueden atenuar los problemas físicos, psicosociales y espirituales de más de $90 \%$ de los enfermos, además de consi- derárseles una necesidad humanitaria (De la Garza y Juárez, 2014).

Para su implementación, se han usado como referentes trabajos como el de Hilbert (1990), que promueve el apoyo social, pues se le supone un constructo multidimensional del cual emergen la red social, la interacción que existe entre los individuos inmersos en dicha red, los recursos que provee la fuente de soporte, la percepción de la persona frente al mismo y los aspectos emocionales e instrumentales que entran en juego. Es así que los cuidados paliativos trabajan con el entorno social del paciente, pues es ahí donde se toman decisiones y se llevan a cabo acciones situadas fuera del terreno médico en el que el enfermo significa y da sentido a su padecimiento (González, Tinoco y Benhumea, 2011). Un ejemplo de ello es que las mujeres con cáncer de mama encuentran en su entorno social más cercano una fuente que genera seguridad, bienestar, tranquilidad y satisfacción en el proceso que viven (Martínez, 2018); no obstante, tales efectos pueden aplicarse a enfermos con otros tipos de cáncer y a ambos sexos.

Por ello, la pareja ha asumido un papel protagónico en el tratamiento del cáncer, siendo así que Wang et al. (2017) concluyen que el estado civil puede influir en el pronóstico de la enfermedad en las personas con cáncer renal, aumentando así la probabilidad de supervivencia en virtud de que los pacientes casados tienen un acceso más expedito al apoyo social y psicológico, aminorando así su angustia, ansiedad y estrés. Por su parte, Berríos y Rivero (2015) encontraron en su estudio dirigido a pacientes con cáncer prostático que la esposa del paciente tenía una influencia positiva en el bienestar y el tratamiento del varón. Ello confirma que el acompañamiento proporcionado por la pareja en el proceso de salud-enfermedad contribuye al fortalecimiento de los factores emocionales y sociales positivos, lo que ayuda a mantener el equilibrio familiar e individual, contribuyendo a la adaptación del paciente (Baca, 2014; Berríos y Rivero, 2015; Jordá y Saus, 2019; Marín et al., 2013; Pérez, Piqueras e Inglés, 2017). Lo anterior implica que la investigación de la pareja y su influencia en las consecuencias negativas generadas por el cáncer cobre particular relevancia. 
Debido a que el cáncer está estrechamente relacionado con el concepto de muerte, se le considera una "enfermedad tabú" que tiende a aislar al paciente de su entorno (Fundación Salud, Innovación y Sociedad [FSIS], 2003), lo que provoca que enfrente solo los grandes cambios emocionales, conductuales y sociales concomitantes (Cano, 2005), lo que induce un importante estrés emocional, de tal forma que Pérez et al. (2017) aseguran que este es un hecho vital no normativo. Dicho estrés es provocado por distintas causas y en diferentes momentos de la enfermedad, ya que puede estar presente en la etapa de diagnóstico, durante el tratamiento o en el estado de remisión (De Brito, 2003; García-Aguado, 2016). Una vez establecido el diagnóstico, y dependiendo de las estrategias psicológicas de las que disponga el paciente, la evolución de la enfermedad se ve afectada por el estrés que proviene de esas distintas fuentes (De la Garza y Juárez, 2014); en efecto, durante la enfermedad y el tratamiento pueden ocurrir cambios importantes en la apariencia y el funcionamiento del cuerpo, así como sobrevenir problemas emocionales y alteraciones de las actividades cotidianas y del rol familiar, social y laboral, induciendo una modificación de las expectativas y los planes a futuro, y comprometiendo el funcionamiento físico, psicológico, emocional y social que influye en la calidad de vida de las personas.

El estrés es definido como una reacción fisiológica del organismo, resultado de la evaluación cognitiva acerca de un estímulo o situación amenazante, que altera los aspectos internos y externos y la capacidad de sobrellevarlos (Cohen, Kamarck y Mermelstein, 1983; Naranjo, 2009), y una clase particular de relación entre la persona y su ambiente que pone en peligro su bienestar (Lazarus y Folkman, 1991). Cohen, Kessler y Underwood (1997) consideran el estrés como un proceso que comienza con la aparición de una demanda ambiental, la cual, después de ser valorada por el individuo en función de sus capacidades personales para afrontarla, es percibida como amenazante, lo que genera respuestas fisiológicas, afectivas y conductuales que tienen como consecuencia final un riesgo para la salud física y psicológica de dicho individuo. Burgos et al. (2020) lo definen como un trastorno biopsicosocial que afecta actualmente a la población mundial y que es característica de una sociedad globalizada que exige y demanda cada día individuos aptos y capacitados para enfrentar y resolver cada uno de los problemas de índole laboral, social o emocional que se les presenten. Estas secuelas, al coincidir con otros factores de riesgo (edad avanzada, bajo estatus o escaso apoyo social), pueden ocasionar una considerable susceptibilidad a diversas enfermedades y tiempos de recuperación más lentos (Honold, Beyer, Lakes y van der Meer, 2012).

La respuesta de los individuos a las situaciones generadoras de estrés dependerá de la manera en que interpreten los eventos ambientales, evalúen sus propios recursos y la posibilidad de afrontar su entorno (Burgos et al., 2020; Naranjo, 2009; Oros, 2002). Al respecto, Lazarus (2000) señala que los individuos dependen de la percepción que tengan de la situación, ya sea como un reto, ya como una amenaza, pues a partir de ello se valorarán los estresores y la respuesta a estos. Si la persona percibe el agente estresor como un reto, lo convertirá en una oportunidad que incrementará su fuerza y desarrollo personal; sin embargo, si lo percibe como una amenaza, lo verá como una situación que tendrá consecuencias negativas y que implicará daños o desventajas (Ansoleaga, 2015; Escamilla, Rodríguez y González, 2009). Esta última valoración es sumamente importante, pues de ella dependerán las consecuencias que genera el estrés, entre las que se incluyen reacciones tales como hostilidad, irritabilidad, temor, tensión muscular, problemas gastrointestinales, insomnio, vértigo, taquicardia, sudoración excesiva y muchas otras (Jiménez y Cubillos, 2010), las que con el tiempo pueden causar enfermedades como ansiedad, depresión, artritis reumatoide, migraña, calvicie, asma, trombosis, colitis, diabetes y muchas más (Bairero, 2017).

Ante el estrés ocasionado por el cáncer, un factor protector es el entorno psicosocial. De hecho, varios estudios han demostrado la influencia de los aspectos psicosociales en el curso de la enfermedad y encontrado un vínculo entre la adaptación psicosocial y la respuesta a los tratamientos médicos (Cantú y Álvarez, 2009). Una explicación de tales hallazgos es que los factores psicosociales previenen la aparición de los trastor- 
nos mentales y disminuyen el estrés emocional al dar al individuo los recursos que le permitan enfrentar la enfermedad y las consecuencias de los tratamientos (Alvarado et al., 2011; Barroihet, Forjaz y Garrido, 2005).

Específicamente, uno de los factores que puede disminuir el impacto psicológico del estrés del paciente con cáncer es el apoyo otorgado por su pareja sana, que se refiere a la ayuda que durante la interacción cotidiana es provista por esta para lograr que aquel se sienta importante, acompañado y complementado (Nurullah, 2012). Tal ayuda puede ser instrumental (ayuda material, ayuda en tareas físicas y en labores que involucren el desempeño de roles), emocional (empatía, amor, confianza y preocupación), informativa (provisión de información, consejos o sugerencias) y valorativa (utilidad), provenientes de una transacción interpersonal que contribuye a sobrellevar la enfermedad (Hilbert, 1990; Poblete et al., 2015; Teherán et al., 2017).

Dada la importancia de un problema como el cáncer, contar con una red de apoyo (sobre todo de la pareja), los pacientes podrán hallar un refugio en dicha red, consiguiendo de esta manera aliviar los cambios inesperados que se derivan de esta patología y mejorar con ello su calidad de vida (Martínez, 2018). Además, se ha demostrado que una red de apoyo contribuye a generar una respuesta positiva al tratamiento, ayuda a afrontar la enfermedad y favorece el que el enfermo manifieste conductas favorables acerca de sí mismo (Biffi y Mamede, 2010), ya que la vivencia de situaciones adversas, estresantes y dolorosas producidas por la enfermedad se ven contrarrestadas por el cuidado, la ayuda y el respaldo de una persona cercana (Flórez, Sánchez-Aragón y Athié, en prensa). En otras palabras, el apoyo social propicia una buena salud física y mental que protege a las personas de los efectos que causan las enfermedades al mejorar la autoestima, promueve el uso de estrategias de afrontamiento más efectivas y de comportamientos saludables, mejora el estado de ánimo y las habilidades para ayudarlas a superar la adversidad (Rondón y Lugli, 2013); asimismo, el apoyo es un factor de protección que provee los recursos psicológicos necesarios para resistir el estrés (Chi et al., 2011). No obstante, los efectos positivos de dicho apoyo se pueden ver entorpecidos si el propio individuo no tiene la capacidad de utilizarlo, si no le es proporcionado en el momento adecuado o simplemente si no le es satisfactorio (Sánchez-Aragón, en prensa; Verhofstadt, Lemmens y Buysse, 2013).

Otro de los aspectos que sin duda desempeñan un papel fundamental en la vida de la díada romántica es la empatía, definida por Nickerson, Butler y Carlin (2011) como la noción de compartir en forma vicaria un sentimiento, imaginarse a sí mismo en la situación de otra persona o experimentar las emociones que otra persona está viviendo (Sánchez-Aragón, 2016). Especialmente en las relaciones románticas, la empatía se basa en la capacidad de comprender los estados psicológicos y emocionales de los demás, de tal forma que los individuos pueden comunicarse e interactuar de manera efectiva y placentera, al tiempo que predicen comportamientos e intenciones entre sí (Sánchez-Aragón y Martínez, 2015).

De acuerdo con Davis (1996) y Samper (2014), la empatía desencadena distintos procesos que involucran un costo cognitivo para quien la experimenta, provocando respuestas que pueden ser intrapersonales, como la preocupación empática, y no afectivas, como los juicios atribucionales e interpersonales, o la conducta de ayuda, la cual se abordó líneas atrás. Empatizar desempeña un importante papel al momento de razonar o reflexionar sobre una situación problemática, como el cáncer (Martínez, Piqueras e Inglés, 2011), pues se puede abordar desde distintas perspectivas al tenerse en cuenta la experiencia de la otra persona.

Otro aspecto mediador en el proceso de salud-enfermedad ha sido el sexo del individuo y el rol de género que le ha sido asignado (Academia Nacional de Medicina de México [ANMM], 2014; García-Vega, 2011), pues hay factores biológicos que predisponen a las personas a estar sanas o enfermas (p.e., enfermedades que se manifiestan más en varones que en las mujeres, o a la inversa) (Rohlfs, Borrell y Fonseca, 2000), lo que se aprecia claramente en el caso de las mujeres, quienes se exponen a patrones de socialización, roles familiares, obligaciones domésticas, expectativas laborales y tipos de ocupación que generan sobrecarga física y emocional, las cuales explican un estado 
de salud más pobre y una menor expectativa de vida saludable (García-Vega, 2011; Rohlfs, s.f.). En el caso de los varones, el mantenimiento de las relaciones de poder, el ideal de éxito, la competitividad, la falta de socialización para expresar sentimientos, así como algunos padecimientos mentales y físicos, explican el modo de enfermar y tienen gran importancia en las dificultades para diagnosticar problemas afectivos y psicológicos encubiertos por somatizaciones o conductas de riesgo (Bonino, 2000; García-Vega, 2011).

Con base en lo anterior, se buscó examinar la relación que tiene la empatía de la persona sana (A), la satisfacción con el apoyo de la pareja y la capacidad de recibirlo por parte del paciente con cáncer (B) y con el estrés de este último (B), según su sexo.

\section{MÉTODO}

\section{Participantes}

Se utilizó un muestreo no probabilístico por sujetos tipo (Coolican, 2005; Hernández, Fernández y Baptista, 2014) que satisfacían los siguientes criterios de inclusión: ser mexicanos con una edad superior a los 18 años (mayoría de edad en México), con escolaridad mínima de secundaria, que tuvieran una pareja con la que cohabitaran desde al menos un mes antes del estudio, y que uno de los miembros de la misma hubiese sido diagnosticado previamente con cualquier tipo de cáncer por un médico (fuera o no que estuviera bajo tratamiento). Participaron 194 parejas heterosexuales con un año de relación al menos y hasta 53 años $(\mathrm{M}=22.93$, D.E. $=11.51)$, y que cohabitaban desde 8 meses antes y hasta 53 años $(\mathrm{M}=21.34$, D.E. $=11.72$ ).

Las personas con cáncer se distribuyeron de la siguiente manera: 141 mujeres y 53 hombres, con edades de entre 21 y 78 años $(\mathrm{M}=47.98$, D.E. $=10.44)$; con secundaria $(24.7 \%)$, preparatoria $(29.4 \%$ ) y licenciatura (45.9\%); con un tiempo de diagnóstico de cáncer de un mes a 20 años $(\mathrm{M}=3.87$, D.E. $=3.70), 84.5 \%$ de los cuales se hallaba en tratamiento al momento de este estudio. Sus parejas (personas sin cáncer) fueron $53 \mathrm{mu}-$ jeres y 141 hombres, con edades entre los 18 y los 77 años $(M=49.13$, D.E. $=10.37)$, con secundaria $(21.6 \%)$, preparatoria $(34.0 \%)$ y licenciatura $(44.3 \%)$.

\section{Instrumentos}

Escala de Empatía en el Contexto Romántico (Sánchez-Aragón y Martínez, 2015).

Utilizada para evaluar la compresión intelectual e imaginativa de la condición de la pareja sin experimentar los sentimientos de esa persona ni la reacción emocional en respuesta de los sentimientos de la pareja (Mehrabian y Epstein, 1972), esta escala consta de 39 reactivos en un formato tipo Likert de cinco puntos de respuesta que indican el nivel de acuerdo con la afirmación, de 1, "Totalmente en desacuerdo", a 5, "Totalmente de acuerdo". Dichos reactivos se distribuyen en cuatro factores que explican $40.35 \%$ de la varianza (índice $\mathrm{KMO}=$.924; Prueba de Esfericidad de Barlett $=6155.060, \mathrm{gl}=780, \mathrm{p}=.000)$, mismos que son los siguientes: 1) Toma de perspectiva (quince reactivos, $\alpha=.85$ ), con reactivos como "Trato de imaginarme a mí mismo(a) en los zapatos de mi pareja cuando así se requiere", o "Trato de pensar como mi pareja con el fin de ser más sensible a lo que le pasa"; 2) Empatía cognoscitiva de las emociones (diez reactivos, $\alpha=.82$ ), con reactivos como "Sé cuándo mi pareja está enojada" "Percibo cuando alguien no le simpatiza a mi pareja"; 3) Perturbación propia (cinco reactivos, $\alpha=.70$ ), con reactivos como "Me pone nervioso(a) si mi pareja se lastima" o "Me da pesar el ver sufrir a mi pareja", y 4) Compasión empática (nueve reactivos, $\alpha=.78$ ), con reactivos como "Trato de prestar atención a las emociones de mi pareja" o "Soy el primero en enterarme sobre cómo se siente mi pareja".

Escala de Satisfacción con el Apoyo Recibido de la Pareja (ESAReP) (Sánchez-Aragón y Calleja, 2021).

Basada en la Escala de Apoyo Social de Sarason, Levine, Basham y Sarason, 1983), su objetivo es evaluar la satisfacción con el apoyo recibido en el contexto de la relación romántica. Está compuesta por 31 reactivos en formato tipo Likert con cinco 
puntos de respuesta que indican el grado de satisfacción: de 1, "Nada satisfecho", a 5, "Muy satisfecho", reactivos que se distribuyen en dos factores que explican $65.92 \%$ de la varianza: Apoyo en la cotidianidad y Apoyo grave. Cada reactivo comienza con la instrucción "¿Qué tan satisfecho me siento...?". El factor Apoyo en la cotidianidad contiene 25 reactivos $(\alpha=.97)$, como “... con la capacidad que tiene mi pareja de escucharme cuando necesito hablar" o "... con la confianza que me brinda mi pareja para poder hablar abiertamente y con franqueza". El factor Apoyo grave consta de seis reactivos $(\alpha=.87)$, tales como: “... con la ayuda de mi pareja si mi mejor amigo(a) me insultó y me dijo que no quería volver a verme" o "... con la ayuda que mi pareja me brinda cuando la necesito".

\section{Escala de Disposición a Recibir Apoyo Social} (EDaRAS) (Sánchez-Aragón y Calleja, 2021).

Este instrumento evalúa la disposición del individuo a recibir apoyo de parte de su pareja. Se encuentra conformada por siete reactivos en formato tipo Likert de cinco puntos que indican frecuencia (de 1, "Nunca hago esto", a 5, "Siempre hago esto). La prueba contiene un único factor que explica $44.59 \%$ de la varianza, y una confiabilidad total, medida por el coeficiente alfa de Cronbach, de .79. "Pido ayuda a otras personas cuando sé que no puedo hacer algo solo" o "Permito que mi familia y amigos me apoyen cuando me encuentro en problemas", son algunos de sus reactivos.

\section{Escala de Estrés Percibido}

(Flórez y Sánchez-Aragón, 2020).

Tiene como objetivo evaluar las percepciones que se tienen respecto al estrés experimentado, en términos de la amenaza percibida por la magnitud del estímulo y las capacidades para hacerle frente. Está compuesta por trece reactivos en formato tipo Likert con cinco opciones de respuesta que indican los grados de frecuencia: de 1, "Nunca", a 5, "Siempre"), distribuidos en dos factores: Descontrol y Control, que explican 50.96\% de la varianza (con un índice KMO de .843, Prueba de esfericidad de Barlett de 1102.686, 78 gl y $p$ de .000). El factor Descontrol, contiene seis reactivos $(\alpha=.77)$, como "¿Qué tan frecuentemente usted se ha sentido ner- vioso(a) y estresado(a)?” o “¿Qué tan frecuentemente usted se ha enojado por cosas que estaban fuera de su control?". El factor Control consta de siete reactivos $(\alpha=.72)$, con reactivos como "¿Qué tan frecuentemente usted lidió exitosamente con problemas irritantes de la vida?" o "¿Qué tan frecuentemente usted fue capaz de controlar la manera en la que utiliza su tiempo?".

\section{Procedimiento}

Psicólogas previamente entrenadas aplicaron los instrumentos antes mencionadas en lugares públicos y privados, como hospitales, clínicas, albergues y oficinas diversas de la Ciudad de México en los que pudieran encontrar participantes que cumplieran con los criterios de inclusión. Todos los participantes aceptaron colaborar de manera libre, voluntaria y anónima, auxiliándoles en caso de que no comprendieran las instrucciones o los reactivos. Se cumplieron las reglas establecidas por la Secretaría de Salud, el reglamento de la Ley General de Salud en Materia de Investigación, la Declaración de Helsinki de 1975, enmendada en 1983, sobre los principios éticos para la investigación médica con humanos, y el Código Ético del Psicólogo.

\section{Análisis de datos}

Para cumplir con el objetivo de esta investigación, se efectuó en primera instancia un análisis de Kolmogrov-Smirnov a fin de explorar si los datos presentaban una distribución normal y decidir acerca de los análisis estadísticos a realizar (paramétricos o no paramétricos). Con base en ello, se decidió llevar a cabo una correlación de Spearman entre el estrés y las variables de empatía, apoyo social y capacidad para recibir apoyo. Dichos cálculos se hicieron utilizando el programa SPSS, v. 23.

\section{RESULTADOS}

Con el fin de responder al objetivo de la investigación, se ejecutó en primer lugar un análisis de Kolmogorov-Smirnov para explorar la distribución de los datos, que en ninguno de los casos, excepto 
en uno, mostró tener una distribución normal, es decir, con valores de $p<0.05$ : empatía, $p=.000$; empatía cognoscitiva de las emociones, $p=.000$; perturbación propia, $p=.000$; compasión empática, $p=.000$; satisfacción con el apoyo (apoyo cotidiano); $p=.000$; apoyo grave, $p=.000$; capacidad de recibir apoyo, $p=.013$; descontrol, $p=.008$, y control, $p=.074$. Por lo anterior, los análisis estadísticos efectuados se basaron en dichos resultados.

Se llevó a cabo una correlación de Spearman entre las variables de empatía de A1 (hombre sano), la satisfacción con el apoyo recibido y la disposición a recibir apoyo de B1 (mujer con cáncer), con el estrés de B1. Los resultados arrojaron coeficientes de bajos a medios, que muestran que, en la medida en que el enfermo con cáncer (B1) se sienta satisfecho ante el apoyo otorgado por su pareja (A1) en el ámbito cotidiano, como escuchar las complicaciones del día a día (apoyo cotidiano), mostrará una disminución de la sensación de nerviosismo o "estrés" y de las reacciones emocionales negativas.

Por otro lado, las sensaciones de autoconfianza y autoeficacia al afrontar y dominar las situaciones estresantes (control del estrés) de B1 se ven favorecidas por el apoyo cotidiano y por la ayuda de la pareja en momentos de necesidad (apoyo grave), como cuando el miembro enfermo tiene algún altercado o experimenta situación especial. Por ende, la disposición de B1 a recibir el apoyo de su pareja sana tiene un efecto positivo sobre su propio control y en la resolución efectiva de los problemas (control del estrés) (Tabla 1).

Tabla 1. Correlación de la empatía de A1, la satisfacción con el apoyo recibido de la pareja y la disposición a recibir apoyo y el estrés de B1.

\begin{tabular}{|c|c|c|c|c|}
\hline \multirow{2}{*}{\multicolumn{3}{|c|}{ Factores }} & \multirow{2}{*}{\multicolumn{2}{|c|}{$\frac{\text { B1 (mujer con cáncer) }}{\text { Estrés }}$}} \\
\hline & & & & \\
\hline & & & Descontrol & Control \\
\hline \multirow{3}{*}{$\begin{array}{l}\text { B1 (mujer } \\
\text { con cáncer) }\end{array}$} & \multirow{2}{*}{ Apoyo } & Apoyo cotidiano & & $.355^{* *}$ \\
\hline & & Apoyo grave & & $.249 * *$ \\
\hline & \multicolumn{2}{|c|}{ Disposición a recibir apoyo } & & $.279^{* *}$ \\
\hline
\end{tabular}

La correlación de Spearman entre las variables de empatía de A2 (mujer sana), la satisfacción con el apoyo recibido y la capacidad de recibir apoyo de B2 (hombre con cáncer) con el estrés de B2 (Tabla 2), arrojó resultados que mostraron una correlación entre el éxito del hombre con cáncer al lidiar con sus problemas y el control de su estrés, con la satis- facción que siente ante la confianza, el consuelo, el cuidado y la compañía (apoyo cotidiano) que le brinda la pareja (A2). Del mismo modo, cuando él se encuentra dispuesto a recibir y pedir el apoyo de ella, su sentimiento de encarar efectivamente sus problemas (control de estrés) se ve favorecido.

Tabla 2. Correlación de la empatía de A2 con la satisfacción con el apoyo recibido de la pareja y la disposición a recibir apoyo de $\mathrm{B} 2$ para el estrés.

\begin{tabular}{|c|c|c|c|c|}
\hline \multirow{3}{*}{ Factores } & \multirow{3}{*}{\multicolumn{2}{|c|}{ Factores }} & \multirow{2}{*}{\multicolumn{2}{|c|}{$\frac{\text { B2 (hombre con cáncer) }}{\text { Estrés }}$}} \\
\hline & & & & \\
\hline & & & Descontrol & Control \\
\hline \multirow{2}{*}{$\begin{array}{l}\text { B2 (hombre } \\
\text { con cáncer) }\end{array}$} & Apoyo & Apoyo cotidiano & & $.295^{*}$ \\
\hline & \multicolumn{2}{|c|}{ Capacidad de recibir apoyo } & & $.384 * *$ \\
\hline
\end{tabular}




\section{DISCUSIÓN}

El cáncer es indudablemente una situación estresante que, tal y como apuntan Moreno, Lara y Alvarado (2014), conlleva un cambio drástico en el estilo de vida de quienes lo padecen y en el de sus familias. Sin embargo, la empatía y el apoyo de quienes rodean a la persona enferma pueden amortiguar los efectos adversos de la enfermedad, como el estrés, sobre todo si provienen de su círculo más cercano, en una relación de igualdad y reciprocidad mediada por un vínculo socioemocional como la pareja (Berndt y Savin-Williams, 1993; Claes, 1998; Collins y Gunnar, 1990).

Para obtener los más altos niveles de salud, las políticas sanitarias deben reconocer que las mujeres y los hombres, debido a sus diferencias biológicas y a sus roles de género, tienen diferentes necesidades, afrontan diversos obstáculos y gozan de distintas oportunidades (OMS, 2008). Lo anterior se ve reflejado en la diferencia que hay entre las Tablas 1 y 2; por consiguiente, en la primera es notable un mayor número de correlaciones con el aumento del control del estrés, mientras que en el segundo solo se aprecian dos correlaciones significativas con el control del estrés. Esto se debe principalmente a la ideología del rol que debe tener cada uno de los sexos, pues la mujer ha sido vista como la cuidadora por excelencia, y el hombre como el agente protector. El mayor número de correlaciones que se observa en la Tabla 1 se puede deber al hecho de que el hombre adopta un nuevo papel en el que la mujer se ve aliviada de sus responsabilidades domésticas y como cuidadora de la familia al ser apoyada (ANMM, 2014; Biffi y Mamede, 2010; Castañeda, 2007; García-Vega, 2011; Rohlfs, s.f.), lo que se ve reflejado en las relaciones entre la satisfacción del apoyo cotidiano, el apoyo grave y la capacidad de recibir apoyo. A su vez, el hombre puede no percibir una mayor satisfacción con el apoyo recibido pues se convierte en una normalidad que puede ser invisibilizada (Peredo, 2003) y no resultar significativa en momentos estresantes, además de que se le asignan atributos como autonomía, autoconfianza, asertividad, orientación al logro e instrumentalidad, lo que hace más difícil que acepte y exprese sentimientos de debilidad, incompetencia y miedo (Matud, 2004; Matud, Guerrero y Matías, 2006).

La satisfacción con el apoyo cotidiano y el apoyo grave fue una variable que resultó significativa para el control del estrés, lo que, como se señaló líneas arriba, puede deberse a que el apoyo social en general salvaguarda a las personas de los efectos que causan las enfermedades (Harden, Northouse y Mood, 2006; Moreno et al., 2014; Rondón y Lugli, 2013) porque mejora la autoestima, promueve el uso de estrategias de afrontamiento más efectivas y de comportamientos saludables, mejora el estado de ánimo y las habilidades para superar la adversidad (Rondón y Lugli, 2013), lo que, de acuerdo con Chi et al. (2011) provee los recursos necesarios para afrontar el estrés.

Del mismo modo, y tal como afirman Dellafiore et al. (2018), la disposición a aceptar el apoyo conyugal afecta la gestión de la enfermedad ante situaciones estresantes en virtud de que contar con aquel permite que la persona con cáncer se sienta cuidada, amada y valorada, mitigando así el efecto de los estresores (García-Torres, García-Méndez, M., y Rivera-Aragón, 2017). Es factible que lo anterior se deba a que la capacidad de recibir apoyo se relaciona directamente con la prosperidad, que otorga al individuo una sensación de tener una vida útil y significativa (Flórez et al., en prensa; Sánchez-Aragón, 2018).

En conclusión, las parejas experimentan diversas alteraciones en la relación cuando uno de los miembros padece cáncer, como cambios en los roles y las responsabilidades, lo que modifica su relación. Sin embargo, enfrentar tal problema unidos puede fortalecer la relación, su amor y compromiso, y descubrir así que su relación es más importante de lo que suponían.

En los resultados del presente estudio fue posible apreciar que la satisfacción con el apoyo brindado por la pareja y la disposición a recibirlo por parte de la persona enferma fueron significativas pero débiles en su relación con el control del estrés, por lo que se considera necesario proseguir investigando las aportaciones de la pareja en el proceso de salud-enfermedad de pacientes con enfermedades crónicas como el cáncer, debido a que pueden contribuir a mejorar su calidad de vida. 


\section{AGRADECIMIENTOS}

Proyecto de investigación, evaluado, avalado y financiado por la Dirección General de Apoyo al Personal Académico de la Universidad Nacional Autónoma de México PAPIIT IN304919 "Factores protectores y de riesgo a la salud en parejas sanas y con enfermedades crónico-degenerativas".

Citación: Santiago-Silva, D.Z. y Sánchez-Aragón, R. (2021). La empatía y el apoyo de la pareja como factores protectores del estrés en pacientes con cáncer. Psicología y Salud, Número Especial, 85-96. https://doi.org/10.25009/pys.v31i3.2730.

\section{REFERENCIAS}

Academia Nacional de Medicina de México (2014). El hombre y la mujer enferman en forma diferente. Revista de la Facultad de Medicina, 57(2), 53-56.

Alvarado A., S., Ochoa C., F.J., Guerra C., H.G., Mulier R., Y.A., Galindo V., O. y Zapata, I., M.R. (2011). Adherencia terapéutica del paciente con cáncer; algunos factores: (perspectiva del oncólogo). Gaceta Mexicana de Oncología, 10(3), $136-142$.

American Cancer Society (2016). ¿Qué es el cáncer? Atlanta, GA: ACS. Recuperado de https://www.cancer.org/content/dam/ CRC/PDF/Public/6041.96.pdf.

Ansoleaga, E. (2015). Indicadores de salud mental asociados a riesgo psicosocial laboral en un hospital público. Revista Médica de Chile, 143(1), 47-55. Doi: 10.4067/S0034-98872015000100006.

Baca R., D. (2014). Resiliencia y apoyo social percibido en pacientes oncológicos que acuden a un hospital de Trujillo. Revista Peruana de Psicología y Trabajo Social, 3(1), 107-124.

Bairero A., M. (2017). El estrés y su influencia en la calidad de vida. Multimed, 21(6), 971-982. Recuperado de http://www.revmultimed.sld.cu/index.php/mtm/article/view/688.

Barroihet D., S., Forjaz, M.J. y Garrido L., E. (2005). Conceptos, teorías y factores psicosociales en la adaptación al cáncer. Actas Españolas de Psiquiatría, 33(6), 390-397.

Berndt, T.J. y Savin-Williams, R.C. (1993). Peer relations and fiendships. En P. H. Tolan y B. J. Cohler (Comps.): Clinical research and practice with adolescents (pp. 203-219). New York: John Wiley \& Sons.

Berríos, R. y Rivero V., A. (2015). El cáncer prostático en la experiencia de pareja: La mujer como guardiana de la salud. Interamerican Journal of Psychology, 49(3), 387-398.

Biffi, R.G. y Mamede, M.V. (2010). Perception of family functioning among relatives of women who survived breast cancer: Gender differences. Revista Latino-Americana de Enfermagem, 18(2), 269-277. Doi: 10.1590/S0104-11692010000200019.

Bonino, L. (2000). Varones, género y salud mental: deconstruyendo la "normalidad" masculinidad. En M. Segarra y A. Carabí (Eds.): Nuevas masculinidades (pp. 41-64). Madrid: Icaria Editorial.

Burgos A., D.J., Rendón L., V.M., Díaz N., C.L., Aguirre C., J.T., Peralta Z., G.A. y Angulo L., T.A. (2020). La relajación y estrés laboral en el personal docente: una revisión sistemática. Lecturas: Educación Física y Deportes, 25(266), 109-123.

Cano V., A. (2005). Control emocional, estilo represivo de afrontamiento y cáncer. Psicooncología, 2(1), 71-80.

Cantú G., R. y Álvarez B., J. (2009). Sucesos vitales y factores psicosociales asociados: el caso de pacientes con cáncer. Psicología y Salud, 19(1), 21-32.

Castañeda A., I.E. (2007). Reflexiones teóricas sobre las diferencias en salud atribuibles al género. Revista Cubana de Salud Pública, 33(2), 1561-3127.

Chi, P., Tsang, S.K.M., Chan, K.S., Xiang, X., Yip, P.S.F., Cheung, Y.T. y Zhang, X. (2011). Marital satisfaction of Chinese under stress: Moderating effects of personal control and social support. Asian Journal of Social Psychology, 14, 15-25. Doi: 10.1111/j.1467-839X.2010.01322.x.

Cohen, S., Kamarck, T. y Mermelstein, R. (1983). A global measure of perceived stress. Journal of Health and Social Behavior, 24(4), 385-396. Doi: 10.2307/2136404.

Cohen, S., Kessler, R. y Underwood, L. (1997). Strategies for measuring stress: studies of psychiatric and physical disorders. En S. Cohen y R. Kessler (Eds.): Measuring stress: A guide for health and social scientist (pp. 3-26). Oxford, UK: Oxford University Press.

Collins, W.A. y Gunnar, M.R. (1990). Social and personality development. Annual Review of Psychology, 41(1), 387-416. Doi: 10.1146/annurev.ps.41.020190.002131. 
Claes, M. (1998). Adolescent's closeness with parents, siblings, and friends in three countries: Canada, Belgium, and Italy. Journal of Youth and Adolescence, 27, 165-184. Doi: 10.1023/A:1021611728880.

Coolican, H. (2005). Métodos de investigación y estadística en psicología. México: El Manual Moderno.

Davis, M.H. (1996). A social psychological approach. Boulder, CO: Westview Prees.

De Brito S., C.S.V. (2003). Representaçao cognitive e emocional, estratégias de coping e qualidade de vida no doente oncológico e familia. Tesis inédita de doctorado. Lisboa: Universidade do Porto. Recuperado de https://repositorio-aberto.up.pt/ handle/10216/22948.

De la Garza S., J.G. y Juárez S., P. (2014). El cáncer. La Ciencia a tu Alcance. Monterrey (México): UANL. Recuperado de http:// eprints.uanl.mx/3465/1/El_Cancer.pdf.

Dellafiore, F., Rosa, D., Pittella, F., Caruso, M.P., Allemano, M. y Caruso, R. (2018). The impact of type 2 diabetes diagnosis on married couples. Journal of Diabetes Nursing, 22, 1-5.

Escamilla, M., Rodríguez, I. y González, M.G. (2009). El estrés como amenaza y como reto: un análisis de su relación. Ciencia y Trabajo, 11(32), 96-101.

Flórez R., Y.N., y Sánchez-Aragón, R. (2020). El estrés (experiencia y percepción de reto vs. amenaza) y la rumia como factores de riesgo a la salud. Salud y Administración, 7(20), 17-27.

Flórez R., Y.N., Sánchez-Aragón, R. y Athié A., A.J. (en prensa). The importance of social and the ability it receive it in subjective well-being and the quality of life of the diabetic patient. En Diabetes: An approach from the social psychology of health. New York: Springer.

Fundación Salud, Innovación y Sociedad (2003). Necesidad y demanda del paciente oncológico. Barcelona: FSIS. Recuperado de http://www.fbjoseplaporte.org/docs/repositori/070517121920.pdf.

García A., A. (2016). Apoyo social percibido, estado emocional y estrés en cáncer de mama tras cirugía. Tesis inédita de maestría. Elche (España): Universidad Miguel Hernández. Recuperado de http://193.147.134.18/bitstream/11000/5725/1/GARCIA_AGUADO_ANAIS_TFM.pdf.

García T., M., García M., M. y Rivera-Aragón, S. (2017). Apoyo social en adultos mexicanos: Validación de una escala. Acta de Investigación Psicológica, 7, 2561-2567. Doi: 10.1016/j.aipprr.2017.02.004.

García V., E. (2011). Acerca del género y la salud. Papeles del Psicólogo, 32(3), 282-288.

González G., N., Tinoco G., A.M. y Benhumea G., L.E. (2011). Salud mental y emociones en pacientes con enfermedades crónico-degenerativas. Un acercamiento a la diabetes mellitus tipo 2. Espacios Públicos, 14(32), 258-279.

Harden, J.K., Northouse, L.L. y Mood, D.W. (2006). Qualitative analysis of couples' experience with prostate cancer by age cohort. Cancer Nursing, 29(5), 367-377. Doi: 10.1097/00002820-200609000-00004.

Hernández, R., Fernández, C. y Baptista, P. (2006). Metodología de la investigación. México: McGraw-Hill Interamericana Educación.

Hilbert, G.A. (1990). Social support in chronic illness. En O. L.Strickland y C. F. Waltz (Eds.): Measurement of nursing outcomes: Measuring client self-care and coping skills (pp.79-96). New York: Springer.

Honold, J., Beyer, R., Lakes, T. y van der Meer, E., (2012). Multiple environmental burdens and neighborhood-related health of city residents. Journal of Environmental Psychology, 32, 305-317. Doi: 10.1016/j.jenvp.2012.05.002.

INFO Cáncer (2019). El cáncer en el mundo y México. Recuperado de https://www.infocancer.org.mx/?c=conocer-el-cancer\&a $=$ estadisticas-mundiales-y-locales.

Instituto Nacional de Estadística y Geografía (2018). Estadísticas a propósito del Día Mundial contra el Cáncer (4 de febrero. Ciudad de México: INEGI. Recuperado de https://www.infocancer.org.mx/?c=cancer-cifras\&a=estadisticas-2018.

Jiménez, A. y Cubillos, R. (2010). Estrés percibido y satisfacción laboral después del terremoto ocurrido el 27 de febrero de 2010 en la Zona Centro-Sur de Chile. Terapia Psicológica, 28(2), 187-192. Doi: 10.4067/S0718-48082010000200007.

Jordá G., C. y Saus O., C. (2019). Efecto del cáncer de mama sobre el ajuste diádico en parejas atendidas en un hospital de Valencia. Nure Investigación, 16(99), 1-10.

Lazarus, R. (2000). Estrés y emoción. Manejo e implicaciones en nuestra salud. Bilbao (Espeña): Desclée de Brouwer.

Lazarus, R. y Folkman, S. (1991). Estrés y procesos cognitivos. Madrid: Ediciones Roca.

Marín K., C.L., Martínez P., D.F., Vera P., F., Echeverri R., S., Muñoz U., D.M., Quelal A. Y.K., Sánchez C., D.M. y Urbano G., S.F. (2013). Calidad de vida en pacientes en tratamiento de cáncer de mama, Pereira, Colombia 2010. Investigaciones Andinas, 15(27), 811-823.

Martínez A., E., Piqueras J., A. e Inglés C., J. (2011). Relaciones entre inteligencia emocional y estrategias de afrontamiento ante el estrés. Revista Electrónica de Motivación y Emoción, 37, 20-21.

Martínez R., A.M. (2018). Calidad de vida y apoyo social en mujeres con cáncer de mama. Ciencia y Cuidado, 15(1), 34-47. Doi: $10.22463 / 17949831.1232$. 
Matud, M.P. (2004). Gender differencer in stress and coping styles. Personality and Individual Differences, 37(2004), 1401-1415. Doi: 10.1016/j.paid.2004.01.010.

Matud, M.P., Guerrero, K., y Matías, R.G. (2006). Relevancia de las variables sociodemográficas en las diferencias de género en depresión. International Journal of Clinical and Health Psychology, 6(1), 7-21.

Mehrabian, A. y Epstein, N. (1972). A measure of emotional empathy. Journal of Personality, 40, 525-543.

Moreno L., S.L., Lara M., F.U. y Alvarado A., S. (2014). Impacto psicológico en la pareja de la paciente con cáncer de mama. Gaceta Mexicana de Oncología, 13(1), 47-52.

Naranjo M., L. (2009). Una revisión teórica sobre el estrés y algunos aspectos relevantes de éste en el ámbito educativo. Educación, 33(2), 171-190.

National Cancer Institute (2015). What is cancer? Bethesda, MD: NCI. Recuperado de https://www.cancer.gov/about-cancer/ understanding/what-is-cancer.

Nickerson, R.S., Butler, S.F. y Carlin, M. (2011). Empathy and knowledge projection En J. Decety y W. Ickes (Eds.): The Social Neuroscience of Empathy (pp. 43-56). Cambridge, MA: The MIT Press.

Nurullah, A. (2012). Received and provided social support: a review of current evidence and future directions. American Journal of Health Studies, 27(3), 173-188.

Organización Mundial de la Salud (2008). Subsanar las desigualdades en una generación. Alcanzar la equidad sanitaria actuando sobre los determinantes sociales de la salud. Ginebra: OMS. Recuperado de https://apps.who.int/iris/bitstream/ handle/10665/69830/WHO_IER_CSDH_08.1_spa.pdf?sequence=1\&isAllowed=y.

Organización Mundial de la Salud (2016). Cáncer. Ginebra: OMS. Recuperado de https://www.who.int/cancer/es/.

Organización Mundial de la Salud (2017). 10 datos sobre el cáncer. Ginebra: OMS. Recuperado de https://www.who.int/features/ factfiles/cancer/es/.

Organización Mundial de la Salud (2018). Cáncer. Datos y cifras. Ginebra: OMS. Recuperado de https://www.who.int/es/newsroom/fact-sheets/detail/cancer.

Organización Panamericana de la Salud y Organización Mundial de la Salud (2013). Salud, ambiente y desarrollo sostenible: hacia el futuro que queremos. Washington, D.C.: OPS/OMS. Recuperado de https://iris.paho.org/bitstream/handle/10665.2/3472/ Salud,\%20ambiente\%20y\%20desarrollo\%20sotenible\%20hacia\%20el\%20futuro\%20que\%20queremos\%20sDE.pdf.

Oros S., L.B. (2002). Estrés y variables moderadoras de la percepción de la amenaza. Interdisciplinaria, 19(2), 159-183.

Peredo B., E. (2003). Mujeres, trabajo doméstico y relaciones de género: reflexiones a propósito de la lucha de las trabajadoras bolivianas. En M. León T. (Comp.): Mujeres y trabajo: cambios impostergables (pp. 54-65). Ciudad Caucel: Veraz Comunicação. http://biblioteca.clacso.edu.ar/clacso/gt/20101012022000/7pereda.pdf.

Pérez, P., González, A., Mieles, I., y Uribe A., F. (2017). Relación del apoyo social, las estrategias de afrontamiento y los factores clínicos y sociodemográficos en pacientes oncológicos. Pensamiento Psicológico, 15(2), 41-54. Doi: 10.11144/Javerianacali.PPSI15-2.rase.

Poblete, F., Glasinovic, A., Sapag, J., Barticevic, N., Arenas, A. y Padilla, O. (2015). Apoyo social y salud cardiovascular: adaptación de una escala de apoyo social en pacientes hipertensos y diabéticos en la atención primaria chilena. Atención Primaria, 47(8), 523-531. Doi: 10.1016/j.aprim.2014.10.010.

Puente, J. y De Velasco, G. (2019). ¿Qué es el cáncer y cómo se desarrolla? Madrid: Sociedad Española de Oncología Médica (SEOM). Recuperado de https://seom.org/informacion-sobre-el-cancer/que-es-el-cancer-y-como-se-desarrolla?showall=1.

Rohlfs, I. (s.f.). Género y salud: diferencias y desigualdades. Recuperado de http://quark.prbb.org/27/027036.htm.

Rohlfs, I., Borrell, C. y Fonseca, M.C. (2000). Género, desigualdades y salud pública: conocimientos y desconocimientos. Gaceta Sanitaria, 14(S3), 60-71.

Rondón, J. y Lugli, Z. (2013). Efectos del tipo de diabetes, estrategias de afrontamiento, sexo y optimismo en el apoyo social. Revista de Psicopatología y Psicología Clínica, 18(3), 193-207.

Samper G., P. (2014). Diferentes tendencias prosociales: el papel de las emociones. Revista Mexicana de Investigación en Psicología, 6(2), 177-185.

Sánchez-Aragón, R. (2016). La pareja romántica en México. Desentrañando su naturaleza comunicativa y emocional. Ciudad de México: Universidad Nacional Autónoma de México.

Sánchez-Aragón, R. y Calleja, N. (2021). Adaptación y validación de la Escala de Satisfacción con el Apoyo Recibido y diseño y validación de la Escala de Disposición a recibir Apoyo Social. Revista Costarricense de Psicología, 40(1), 37-59. Doi: 10.22544/rcps.v40i01.03.

Sánchez-Aragón, R. y Martínez P., M. (2015). Empatía en el contexto romántico: diseño y validación de una medida. Universitas Psychologica, 15(1), 19-28. Doi: 10.11144/Javeriana.upsy15-1.ecrd.

Sánchez-Aragón, R. (en prensa). Apoyo de la pareja: Satisfacción, capacidad para recibirlo y resiliencia en México. Revista Costarricense de Psicología. 
Sarason, I.G., Levine, H.M., Basham, R.B. y Sarason, B.R. (1983). Assessing social support: The Social Support Questionnaire. Journal of Personality and Social Psychology, 44(1), 127-139. Doi: 10.1037/0022-3514.44.1.127.

Teherán, A., Mejía, M., Álvarez, L., Muñoz, Y., Barrera, M. y Cadavid, V. (2017). Relación entre el apoyo social y las complicaciones agudas de la diabetes tipo 2: un estudio de corte transversal. Revista de Ciencia de la Salud, 15(2), 211-222. Doi: 10.12804/revistas.urosario.edu.co/revsalud/a.5757.

Verhofstadt, L.L.L., Lemmens, G.M.D. y Buysse, A. (2013). Support-seeking, support-provision and support-perception in distressed married couples: A multi-method analysis. Journal of Family Therapy, 35(3), 320-339. Doi: 10.1111/14676427.12001.

Wang, H., Wang, L., Kabirov, I., Peng, L., Chen, G., Yang, Y., A, Z.A. y Xu, W. (2017). Impact of marital status on renal cancer patient survival. Oncotarget, 26(41), 70204-70213. Doi: 10.18632/oncotarget.19600. 